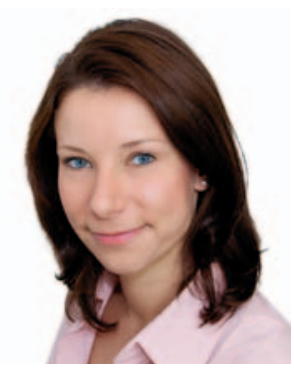

\title{
Nutzen der Positivdrucktherapie für OSA-Patienten: Viele Fragen bleiben offen
}

\section{Wiebke Dohrn}

Überörtliche fachübergreifende Gemeinschaftspraxis für Pneumologie, Allergologie, Schlafmedizin, Gastroenterologie, Innere Medizin und Allgemeinmedizin, Münster

Abstractübersetzung aus Randerath WJ, Treml M, Priegnitz C, et al.: Parameters of overnight pulse wave under treatment in obstructive sleep apnea. Respiration 2016;92:136-143.

Parameter der nächtlichen Pulswellenanalyse bei der Behandlung der obstruktiven Schlafapnoe

\section{Schlüsselwörter}

Pulswellendämpfung · Kardiovaskuläre Risikobewertung · Obstruktive Schlafapnoe · Kontinuierlicher positiver Atemwegsdruck · Pulsausbreitungszeit .

Pulsfrequenzbeschleunigung · Autonomer Zustand

\section{Zusammenfassung}

Hintergrund: Schlafbezogene Atmungsstörungen können zu kardiovaskulären (CV) Erkrankungen beitragen. Für die Behandlung der obstruktiven Schlafapnoe (OSA) wurde ein neuartiger und differenzierter Ansatz zur Analyse der nächtlich photoplethysmographisch aufgezeichneten Pulswellen evaluiert, der die Risikobewertung und die Messung verschiedener Pulswellencharakteristika umfasst.

Ziele: Zweck dieser Studie war es, zu untersuchen, ob und welche der differenzierten Pulswellencharakteristika von der OSA-Behandlung mit positivem Atemwegsdruck (PAP) beeinflusst werden könnten.
Methoden: Die Studie umfasste zwei Protokolle. In der FallKontroll-Studie (Gruppe A) wurden von Pulswellen abgeleitete CV-Risikoindizes, die während der PAP-Therapie aufgezeichnet wurden, mit denen von Patienten mit unbehandelter OSA verglichen, die nach Alter, Body-Mass-Index und CV-Risikoklassen parallelisiert waren ( $n=67 / 67$ ). In der prospektiven PAP-Behandlungsstudie (Gruppe B) nahmen 17 unselektierte Patienten an Schlaftests über die ganze Nacht teil und wurden bei Behandlungsbeginn und nach $23 \pm 19$ Behandlungswochen analysiert. Ergebnisse: Bei den unbehandelten OSA-Patienten (Gruppe A) war die hypoxische Belastung über Nacht erhöht (SpO2-Index $38,7 \pm 17,5$ vs. $24,0 \pm 11,1, p<0,001$ ) und der Pulswellendämpfungsindex (PWA-I) niedriger $(29,4 \pm 9,2$ vs. $33,5 \pm 11,8, p=0,022)$ als bei den behandelten Patienten. In Gruppe B reduzierte die PAPTherapie die hypoxische Belastung und erhöhte den PWA-I signifikant. Der kombinierte CV-Risikoindex war geringfügig, jedoch nicht signifikant reduziert.

Schlussfolgerungen: Die PAP-Therapie modifizierte die hypoxische Belastung und die von Pulswellen abgeleiteten Marker. Der PWA-I - der mit dem sympathischen Gefäßtonus assoziiert ist wurde am stärksten durch PAP modifiziert. Dieser neuartige Ansatz für Marker der CV-Funktion sollte in prospektiven Studien weiter evaluiert werden.

(c) 2017 S. Karger GmbH, Freiburg

\section{KARGER}

Fax +497614520714 information@karger.com www.karger.com
() 2017 S. Karger GmbH, Freiburg 


\section{Transfer in die Praxis}

\section{Hintergrund}

Seit Veröffentlichung der Studienergebnisse des SAVE-Trials [1] ist eine große Unsicherheit hinsichtlich der Therapieindikation bei obstruktiver Schlafapnoe (OSA) entstanden. In dieser großen multizentrisch-randomisierten Studie konnte keine Reduktion der Mortalität oder kardiovaskulärer Ereignisse durch eine Add-onPositivdrucktherapie bei Patienten mit einer OSA nachgewiesen werden. Die Aussagekraft der Resultate wird zwar durch die eingeschränkte Compliance mit geringen Nutzungszeiten im SAVETrail sowie die unausgeglichene Geschlechterverteilung und den eingeschränkten Beobachtungszeitraum diminuiert, dennoch werden zukünftig neue Parameter für den Einsatz der Positivdrucktherapie bei OSA definiert werden müssen.

\section{Studienergebnisse}

Randerath et al. haben in der vorliegenden Studie die Effektivität der Positivdrucktherapie auf verschiedene Pulswellenparameter und die damit verbundenen Einflüsse auf das kardiovaskuläre Risikoprofil untersucht. Die Positivdrucktherapie reduzierte die hypoxische Belastung und beeinflusste auch einzelne Parameter, die von der Hypoxie unabhängig waren (z.B. pulse wave attenuation index, PWA-I) signifikant. Die Verringerung des kombinierten kardiovaskulären Risikoindexes war geringfügig, jedoch nicht signifikant. Damit stellt die Beeinflussung einzelner Pulswellenparameter einen ersten und in Zukunft möglicherweise entscheidenden Parameter zur Beurteilung der Effektivität einer Positivdrucktherapie auf das kardiovaskuläre System dar.

\section{Diskussion}

Um eine differenzierte Aussagekraft zu erzielen, sollten daher weitere Studien unter standardisierten Bedingungen mit großen Patientenzahlen im Langzeitverlauf multivariat durchgeführt werden. Zusammen mit weiteren Parametern kann so definiert werden, wann eine Positivdrucktherapie einen definitiven Nutzen für den OSA-Patienten darstellt. Interessant wäre auch der Ansatz, ob eine Therapie einen kardioprotektiven Effekt erzielt, wenn Patienten symptomatisch im Sinne einer Tagessymptomatik sind und von einem unerholsamen Schlaf auszugehen ist.

Zu klären bleibt auch der Effekt auf die Pulswellenparameter und damit auch das kardiovaskuläre Risiko der Positivdrucktherapie auf weitere schlafbezogene Atmungsstörungen wie die zentrale Schlafapnoe (ZSA) und Hypoventilationssyndrome. Insbesondere die adaptive Servoventilationstherapie (ASV-Therapie) im Rahmen der ZSA ist seit der SERVE-HF-Studie [2] in Verruf geraten. Zu klären bleibt, ob die Positivdrucktherapie einen Nutzen für dieses Patientenklientel darstellt oder ob eher von einem Schaden auszugehen ist. Bei der ZSA könnte mit der Pulswellenanalyse und weiteren Parametern geklärt werden, ob sich ein Effekt auf die Therapie zentraler respiratorischer Ereignisse ergibt, oder ob diese, wie bereits vielfach diskutiert, eher als kardioprotektiv anzusehen sind. Interessant wäre es auch, den Einfluss von Therapiealternativen zur Positivdrucktherapie bei der OSA, wie Unterkieferprotrusionsschienen und Zungengrundschrittmacher, mittels Pulswellenanalyse zu evaluieren. Die Therapieansätze der alternativen Therapieoptionen variieren im Vergleich zur Positivdrucktherapie und bedienen sich einer Erweiterung des Oropharyngealraumes und damit nicht eines Positivdrucks, welcher im Thorax zu Volumenverschiebungen hinsichtlich Blut- und Luftverteilung führt.

\section{Fazit für die Praxis}

Die differenzierte Analyse einzelner Pulswellenparameter kann einen wichtigen Aspekt in Bezug auf das Herz-Kreislauf-System beim Einsatz der Positivdrucktherapie bei OSA darstellen. Sie sollte zusammen mit anderen Parametern im Rahmen größerer, multizentrischer Studien in standardisierter Form eingesetzt werden, um den Therapiebenefit der Positivdrucktherapie auf die OSA neu zu evaluieren. Auch andere schlafbezogene Atmungsstörungen sowie Therapiealternativen sollten hierbei Beachtung finden.

\section{Disclosure Statement}

Hiermit erkläre ich, dass keine Interessenskonflikte in Bezug auf den vorliegenden Kommentar bestehen.

\section{Literatur}

1 McEvoy RD, Antic NA, Heeley E, et al.: CPAP for prevention of cardiovascular events in obstructive sleep apnea. N Engl J Med 2016;375:919-931.

2 Cowie MR, Woehrle H, Wegscheider K, et al.: Adaptive servo-ventilation for central sleep apnea in systolic heart failure. N Engl J Med 2015;373: 1095-105.

Kontaktadresse: Dr. Wiebke Dohrn, Überörtliche fachübergreifende Gemeinschaftspraxis, für Pneumologie, Allergologie, Schlafmedizin, Gastroenterologie, Innere Medizin und Allgemeinmedizin, Schaumburgstrasse 3, 48145 Münster, Deutschland, Wiebke.Dohrn@gmx.de 